in vivo $34: 2845-2850(2020)$

doi:10.21873/invivo.12111

\title{
Prognostic Impact of Coagulation Activity in Patients Undergoing Curative Resection for Pancreatic Ductal Adenocarcinoma
}

\author{
KAZUKI MATSUMURA, HIROMITSU HAYASHI, NORIO UEMURA, LIU ZHAO, \\ TAKAAKI HIGASHI, TAKANOBU YAMAO, FUMIMASA KITAMURA, YUSUKE NAKAO, \\ TOSHIHIKO YUSA, RUMI ITOYAMA, KATSUNORI IMAI, YO-ICHI YAMASHITA and HIDEO BABA \\ Department of Gastroenterological Surgery, Graduate School of Life Sciences, \\ Kumamoto University, Kumamoto, Japan
}

\begin{abstract}
Background/Aim: The aim of this study was to elucidate the clinical impact of coagulation disorders on outcomes after curative resection of pancreatic ductal adenocarcinoma. Patients and Methods: Preoperative coagulation activity in 135 patients, who had undergone curative resections for pancreatic ductal adenocarcinoma was retrospectively evaluated and the impact on survival outcomes analyzed. Results: A prolonged prothrombin timeinternational normalized ratio $(P T-I N R)(\geq 1.1)$ was detected in 23/135 patients (17\%). Univariate analysis that showed prolonged PT-INR was associated with worse relapse-free (hazard ratio=1.79, $p=0.044$ ) and overall (hazard ratio $=2.18, p=0.004)$ survival. Multivariate analyses showed prolonged PT-INR, large tumor (>30 mm), and lymph node metastasis were independent predictors of poor overall survival. Conclusion: Prolonged PT-INR may be a predictor of poor prognosis in patients with pancreatic ductal adenocarcinoma who have undergone curative resection. Coagulation disorders may be a therapeutic target for improving outcomes of pancreatic ductal adenocarcinoma.
\end{abstract}

Pancreatic ductal adenocarcinoma (PDAC) comprises $85 \%$ of pancreatic cancer cases and remains one of the most common and aggressive malignancies, with a 5-year survival rate of approximately $20 \%$ despite improvements in achieving macroscopically curative resection with surgical procedures

This article is freely accessible online.

Correspondence to: Hideo Baba, MD, Ph.D., FACS, Department of Gastroenterological Surgery, Graduate School of Life Sciences, Kumamoto University, 1-1-1 Honjo, Chuo-ku, Kumamoto 8600811, Japan. Tel: +81 963735211, Fax: +81 963714378, e-mail: hdobaba@kumamoto-u.ac.jp

Key Words: Pancreatic ductal adenocarcinoma, coagulation activity, coagulation disorders, PT-INR, prognosis. and adjuvant chemotherapy (1). Several clinical trials have shown that neoadjuvant therapies can prolong survival in patients with PDAC because such therapies can down-stage PDAC and thus improve the curative resection rate. However, it remains to be determined which regimen is the most suitable for PDAC according to each patient's clinical, genetic, and performance status (2). To improve selection of treatment strategies for PDAC and its outcomes, prognostic biomarkers that can be measured easily and reliably are needed to optimally evaluate tumor aggressiveness. A close association between cancer biology and coagulation disorders that has an impact on tumor progression has been reported (3). Hypercoagulability is more likely to occur in patients with pancreatic cancer than in those with other types of cancer (4). Patients with pancreatic cancer are predisposed to developing venous thromboembolic events (VTEs) (5). VTEs in patients with cancer are caused by hypercoagulable states and associated with a poor prognosis (6). Pancreatic resection is also associated with VTEs (7). In patients with hepatocellular carcinoma, coagulation disorders are associated with various complications and poor prognosis (8). Increased fibrinogen, fibrin split products, and D-dimer levels have been associated with worse survival outcomes in patients with breast and colorectal cancer (9-11). In patients with lung cancer, a prolonged prothrombin time-international normalized ratio (PTINR) was found to be associated with worse survival outcomes (12). However, the clinical impact of coagulation disorders in PDAC is still unclear. Therefore, the purpose of this study was to evaluate the prognostic significance of coagulation disorders in patients with PDAC who had undergone curative resection.

\section{Patients and Methods}

Patients. Between October 2010 and March 2019, 157 consecutive patients underwent pancreatectomy for pancreatic cancer at the Department of Gastroenterological Surgery, Kumamoto University. PDAC was histologically confirmed in 144 of them. Nine of these 144 patients were excluded because they were taking anticoagulant 
Table I. Patient characteristics $(n=135)$.

\begin{tabular}{llc}
\hline Characteristic & & Value \\
\hline Age, years & Median (range) & $68.0(36-90)$ \\
Gender, $\mathrm{n}(\%)$ & Male & $81(60 \%)$ \\
BMI, $\mathrm{kg} / \mathrm{m}^{2}$ & Median (range) & $21.5(16.0-29.6)$ \\
CEA, $\mathrm{ng} / \mathrm{ml}$ & Median (range) & $3.5(0.6-18.9)$ \\
CA19-9, U/ml & Median (range) & $56.4(0.6-7313)$ \\
Tumor location, $\mathrm{n}(\%)$ & Head & $88(65.2 \%)$ \\
& Body & $31(22.9 \%)$ \\
& Tail & $16(11.9 \%)$ \\
T-Stage, $\mathrm{n}(\%)$ & T1 & $11(8.1 \%)$ \\
& T2 & $20(14.8 \%)$ \\
& T3 & $106(78.5 \%)$ \\
Tumor size, mm & T4 & $4(3.0 \%)$ \\
N-Stage, $\mathrm{n}(\%)$ & Median (range) & $28(3.0-70)$ \\
& N0 & $58(43.0 \%)$ \\
Operative procedure, $\mathrm{n}(\%)$ & N1 & $77(57.0 \%)$ \\
& SSPPD & $86(63.7 \%)$ \\
& DP & $44(32.6 \%)$ \\
& DP-CAR & $2(1.5 \%)$ \\
& TP & $5(3.7 \%)$ \\
& With PVR & $25(18.5 \%)$ \\
\hline
\end{tabular}

BMI: Body mass index; CEA: carcinoembryonic antigen; CA19-9: carbohydrate antigen 19-9; SSPPD: subtotal stomach-preserving pancreaticoduodenectomy; DP: distal pancreatomy; DP-CAR: distal pancreatomy with en bloc celiac axis resection; PVR: portal vein resection.

therapy. Finally, this study cohort consisted of 135 patients. This study was approved by the Kumamoto University Hospital Ethics Committee (2020-No.1291) and informed consent was obtained from each patient according to the Institutional Review Board protocols. The enrolled patient's characteristics are shown in Table I.

Surgical strategy and postoperative follow-up. At our Institution, the routine surgical strategy for PDAC is pancreatic resection with D2 dissection of the lymph nodes according to tumor location or tumor size, as described previously (13). Gemcitabine and fluorouracilbased chemotherapy was administered as adjuvant chemotherapy from 2004 to 2012, and S-1 after 2013. All patients were monitored either until January 2020 or their death, whichever came first. They were followed-up at 3 -monthly intervals by our hospital or affiliated hospitals. The mean follow-up period was 25 months.

Stage of cancer and cut-off for blood tests. The tumors were staged according to the seventh edition of the American Joint Committee on Cancer/International Union Against Cancer staging manual. Postoperative complications were graded according to the ClavienDindo classification (14). In this report, postoperative complications were defined as those scoring 3 or more in that classification. Blood tests were performed within the month before pancreatic resection. The cut-off value for each clotting factor was based on normal values used at our Institution. For clinical factors for which normal values had not been established, median values were used.

Statistical analysis. Comparisons between groups were examined using the Mann-Whitney $U$-test for continuous variables, and the chisquared or Fisher's exact test for categorical variables. Continuous
Table II. Preoperative coagulation-related variables in 135 patients who underwent curative resection of pancreatic ductal carcinoma.

\begin{tabular}{llc}
\hline Factor & Subgroup & $\mathrm{N}(\%)$ \\
\hline Platelet count & $\geq 15.8 \times 10^{4} / \mu \mathrm{l}$ & $111(82.2 \%)$ \\
& $<15.8 \times 104 / \mu \mathrm{l}$ & $24(17.8 \%)$ \\
PT & $>80 \%$ & $123(91.1 \%)$ \\
& $\leq 80 \%$ & $12(8.9 \%)$ \\
PT-INR & $<1.1$ & $112(83.0 \%)$ \\
& $\geq 1.1$ & $23(17.0 \%)$ \\
APTT & $\geq 80 \%$ & $86(63.7 \%)$ \\
& $<80 \%$ & $49(36.3 \%)$ \\
Supplement (immature data) & & \\
Fibrinogen (n=48) & $\leq 373 \mathrm{mg} / \mathrm{dl}$ & $8(16.7 \%)$ \\
& $>373 \mathrm{mg} / \mathrm{dl}$ & $40(83.3 \%)$ \\
AT-III (n=39) & $>90 \%$ & $34(87.2 \%)$ \\
& $\leq 90 \%$ & $5(12.8 \%)$ \\
TAT (n=38) & $<3.0 \mathrm{ng} / \mathrm{ml}$ & $28(73.7 \%)$ \\
& $\geq 3.0 \mathrm{ng} / \mathrm{ml}$ & $10(26.3 \%)$ \\
D-Dimer $(\mathrm{n}=59)$ & $<1.0 \mu \mathrm{g} / \mathrm{ml}$ & $28(47.5 \%)$ \\
& $\geq 1.0 \mu \mathrm{g} / \mathrm{ml}$ & $31(52.5 \%)$ \\
FDP-E (n=39) & $\geq 5.0 \mathrm{ng} / \mathrm{ml}$ & $27(69.2 \%)$ \\
& $\geq 5.0 \mathrm{ng} / \mathrm{ml}$ & $13(30.8 \%)$ \\
HPT $(\mathrm{n}=17)$ & $<90 \%$ & $12(70.6 \%)$ \\
& $\geq 90 \%$ & $5(29.4 \%)$ \\
\hline
\end{tabular}

APTT: Activated partial thromboplastin time; AT-III: antithrombin III; FDP-E: fibrin/fibrinogen degradation products-E; HPT: hepaplastin test; TAT: thrombin antithrombin III complex; PT: prothrombin time; PT-INR: prothrombin time-international normalized ratio. The cut-off value for each clotting factor was based on normal values used at our Institution.

variables are presented as the median with range and categorial variables as number with percentage. Relapse-free survival (RFS) rate and overall survival (OS) rate were calculated using the KaplanMeier method and compared using the log-rank test. The prognostic factors for RFS and OS after pancreatic resection were investigated using Cox regression models. $p$-Values are two-sided, and $p<0.05$ was considered to denote statistical significance. JMP (version 12; SAS Institute, Cary, NC, USA) was used for all statistical analyses.

\section{Results}

Abnormalities in the coagulation-related variables of platelet count, prothrombin time, PT-INR, and activated partial thromboplastin time were detected in $17.8 \%, 8.9 \%, 17 \%$, and $36.3 \%$ of patients, respectively (Table II). According to univariate Cox regression analysis, male sex [hazard ratio $(\mathrm{HR})=1.89,95 \%$ confidence interval $\quad(\mathrm{CI})=1.20-3.08$; $p=0.006)$, high carbohydrate antigen 19-9 (CA19-9) concentration $(\mathrm{HR}=1.58,95 \% \mathrm{CI}=1.02-2.50 ; p=0.041)$, Tstage $\geq 3 \quad(\mathrm{HR}=2.45,95 \% \mathrm{CI}=1.21-5.88 ; p=0.001)$, large tumor size $(\mathrm{HR}=1.57,95 \% \mathrm{CI}=1.02-2.41 ; p=0.041)$, lymph node metastasis $(\mathrm{HR}=1.94,95 \% \mathrm{CI}=1.25-3.05 ; p=0.003)$, and prolonged PT-INR $(\mathrm{HR}=1.79,95 \% \mathrm{CI}=1.02-2.99$; $p=-0.044)$ were significantly associated with shorter RFS (Table III). Survival curve analysis identified that prolonged PT-INR was 
Table III. Results of univariate and multivariate analyses for relapse-free survival in patients who had undergone curative resection for pancreatic ductal carcinoma.

\begin{tabular}{|c|c|c|c|c|c|c|}
\hline \multirow[t]{2}{*}{ Variable } & \multicolumn{3}{|c|}{ Univariate analysis } & \multicolumn{3}{|c|}{ Multivariate analysis } \\
\hline & $\mathrm{HR}$ & $95 \% \mathrm{CI}$ & $p$-Value & $\mathrm{HR}$ & $95 \% \mathrm{CI}$ & $p$-Value \\
\hline Age $>65$ years & 1.22 & $0.78-1.96$ & 0.381 & & & \\
\hline Male sex & 1.89 & $1.20-3.08$ & 0.006 & 1.82 & $1.15-2.96$ & 0.0098 \\
\hline $\mathrm{CEA}>5.0 \mathrm{ng} / \mathrm{ml}$ & 1.38 & $0.85-2.18$ & 0.187 & & & \\
\hline CA19-9 >37 U/ml & 1.58 & $1.02-2.50$ & 0.041 & & & 0.3217 \\
\hline Blood loss $>700 \mathrm{~g}$ & 1.42 & $0.92-2.19$ & 0.105 & & & \\
\hline Operative time $>500 \mathrm{~min}$ & 1.10 & $0.66-1.76$ & 0.697 & & & \\
\hline T-Stage $\geq 3$ & 2.45 & $1.21-5.88$ & 0.010 & & & 0.0711 \\
\hline Tumor size >30 mm & 1.57 & $1.02-2.41$ & 0.041 & & & 0.2808 \\
\hline Lymph node metastasis & 1.94 & $1.25-3.05$ & 0.003 & 1.87 & $1.21-2.95$ & 0.0048 \\
\hline Complication, $\mathrm{CDC} \geq 3$ & 1.03 & $0.64-1.74$ & 0.903 & & & \\
\hline Platelet count $<15.8 \times 10^{4} / \mu 1$ & 1.00 & $0.61-1.78$ & 0.971 & & & \\
\hline PT $<80 \%$ & 1.61 & $0.71-3.15$ & 0.237 & & & \\
\hline PT-INR $\geq 1.1$ & 1.79 & $1.02-2.99$ & 0.044 & & & 0.0956 \\
\hline APTT $\geq 80 \%$ & 1.08 & $0.69-1.71$ & 0.739 & & & \\
\hline
\end{tabular}

APTT: Activated partial thromboplastin time; CEA: carcinoembryonic antigen; CA19-9: carbohydrate antigen 19-9; CDC: Clavien-Dindo classification; CI: confidence intervaI; HR: hazard ratio; PT: prothrombin time; PT-INR: prothrombin time-international normalized ratio. Bold values indicate statistical significance.
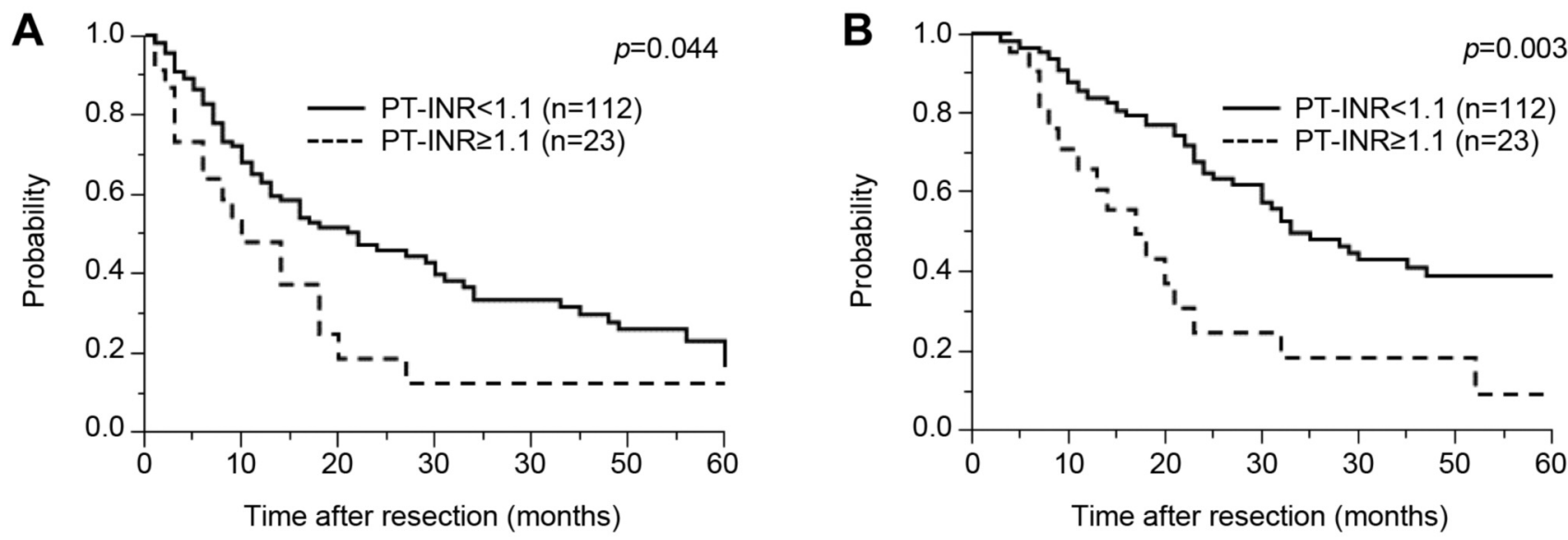

Figure 1. Kaplan-Meier analysis of relapse-free $(A)$ and overall $(B)$ survival according to prothrombin time-international normalized ratio $(P T$ $I N R)$ in patients who underwent curative resection for pancreatic ductal adenocarcinoma (PDAC).

significantly associated with worse RFS compared with normal PT-INR, the median RFS in patients with prolonged PT-INR being significantly shorter than that in patients with normal PT-INR (11 and 22 months, respectively; $p=0.044$ ) (Figure 1A).

According to multivariate Cox regression analyses, male sex $(H R=1.86,95 \% C I=1.15-2.96, p=0.0098)$ and lymph node metastasis $(\mathrm{H}=1.87,95 \% \mathrm{CI}=1.21-2.95 ; p=0.0048)$ were independent predictors of shorter RFS. For OS, male sex $(\mathrm{HR}=1.76,95 \% \mathrm{CI}=1.06-3.01 ; p=0.029)$, high CA19-9 concentration $(\mathrm{HR}=1.78,95 \% \mathrm{CI}=1.07-3.05 ; p=0.027), \mathrm{T}$ stage $\geq 3(\mathrm{HR}=2.52,95 \% \mathrm{CI}=1.04-8.30 ; p=0.041)$, large tumor $(\mathrm{HR}=2.48,95 \% \mathrm{CI}=1.51-4.16 ; p<0.001)$, lymph node metastasis $(\mathrm{HR}=2.37,95 \% \mathrm{CI}=1.42-4.08 ; p=0.001)$, and prolonged PT-INR $(\mathrm{HR}=2.18,95 \% \mathrm{CI}=1.26-3.97 ; p=0.004)$ were significantly associated with shorter OS according to univariate Cox regression analysis (Table IV). According to multivariate Cox regression analyses, large tumor $(\mathrm{HR}=2.22$, 95\% CI=1.34-3.75; $p=0.0018)$, lymph node metastasis $(\mathrm{HR}=2.3,95 \% \mathrm{CI}=1.36-4.00 ; p=0.0016)$, and prolonged PTINR $\quad(\mathrm{HR}=2.65, \quad 95 \% \quad \mathrm{CI}=1.45-4.63 ; \quad p=0.002) \quad$ were independent predictors of worse OS. 
Table IV. Results of univariate and multivariate analyses for overall survival in patients who had undergone curative resection for pancreatic ductal carcinoma.

\begin{tabular}{|c|c|c|c|c|c|c|}
\hline \multirow[t]{2}{*}{ Variable } & \multicolumn{3}{|c|}{ Univariate analysis } & \multicolumn{3}{|c|}{ Multivariate analysis } \\
\hline & HR & $95 \% \mathrm{CI}$ & $p$-Value & HR & $95 \% \mathrm{CI}$ & $p$-Value \\
\hline Age $>65$ years & 1.46 & $0.87-2.53$ & 0.153 & & & \\
\hline Male sex & 1.76 & $1.06-3.01$ & 0.029 & & & 0.2608 \\
\hline CEA $>5.0 \mathrm{ng} / \mathrm{ml}$ & 1.58 & $0.92-2.65$ & 0.097 & & & \\
\hline CA19-9 >37 U/ml & 1.78 & $1.07-3.05$ & 0.027 & & & 0.2464 \\
\hline Blood loss $>700 \mathrm{~g}$ & 1.52 & $0.94-2.49$ & 0.091 & & & \\
\hline Operative time $>500 \mathrm{~min}$ & 1.15 & $0.65-1.94$ & 0.614 & & & \\
\hline T-Stage $\geq 3$ & 2.52 & $1.04-8.30$ & 0.041 & & & 0.1143 \\
\hline Tumor size $>30 \mathrm{~mm}$ & 2.48 & $1.51-4.16$ & $<0.001$ & 2.22 & $1.34-3.75$ & 0.0018 \\
\hline Lymph node metastasis & 2.37 & $1.42-4.08$ & 0.001 & 2.30 & $1.36-4.00$ & 0.0016 \\
\hline Complication $(\mathrm{CDC} \geq 3)$ & 1.15 & $0.66-2.16$ & 0.632 & & & \\
\hline Platelet count $<15.8 \times 10^{4} / \mu 1$ & 1.28 & $0.70-2.19$ & 0.401 & & & \\
\hline PT $<80 \%$ & 2.04 & $0.85-4.20$ & 0.104 & & & \\
\hline PT-INR $\geq 1.1$ & 2.18 & $1.26-3.97$ & 0.004 & 2.65 & $1.45-4.63$ & 0.0023 \\
\hline APTT $\geq 80 \%$ & 1.03 & $0.62-1.74$ & 0.908 & & & \\
\hline
\end{tabular}

APTT: Activated partial thromboplastin time; CEA: carcinoembryonic antigen; CA19-9: carbohydrate antigen 19-9; CDC: Clavien-Dindo classification; CI: confidence intervaI; HR: hazard ratio; PT: prothrombin time; PT-INR: prothrombin time-international normalized ratio. Bold values indicate statistical significance.

Table V. Characteristics of patients according to prothrombin time-international normalized ratio (PT-INR) status.

\begin{tabular}{llccc}
\hline Variable & & $\begin{array}{c}\text { Normal PT-INR } \\
(\mathrm{n}=112)\end{array}$ & $\begin{array}{c}\text { Prolonged PT-INR } \\
(\mathrm{n}=23)\end{array}$ & $p$-Value \\
& & $68.5(40-90)$ & $67(36-79)$ & 0.361 \\
Age, years & Median (range) & $63(56.2 \%)$ & $18(78.2 \%)$ & $\mathbf{0 . 0 4 2}$ \\
Gender, $\mathrm{n}(\%)$ & Male & $3.4(0.4-112)$ & $3.5(0.2-18.9)$ & 0.856 \\
CEA, ng/ml & Median (range) & $56.15(0.6-1735)$ & $62.5(0.7-7313)$ & $\mathbf{0 . 0 2 4}$ \\
CA19-9, U/ml & Median (range) & $582(80-13153)$ & $995(86-3623)$ & 0.868 \\
Blood loss, g & Median (range) & $457.5(120-802)$ & $472(248-840)$ & 0.476 \\
Operation time, min & Median (range) & $90(80.3 \%)$ & $19(82.6 \%)$ & 0.801 \\
T-Stage $\geq 3, \mathrm{n}(\%)$ & Median (range) & $28(3-70)$ & $35(15-65)$ & $\mathbf{0 . 0 2 2}$ \\
Tumor size, mm & Median (range) & $56(50 \%)$ & $13(56.5 \%)$ & 0.056 \\
Lymph node metastasis, $\mathrm{n}(\%)$ & Yes & $25(22.3 \%)$ & $7(30.4 \%)$ & 0.397 \\
Complication $(\mathrm{CDC} \geq 3), \mathrm{n}(\%)$ & Total & $3(2.7 \%)$ & $3(13 \%)$ & 0.056 \\
& Postoperative breeding $(\%)$ & $4(3.6 \%)$ & $0(0 \%)$ & Not available \\
Postoperative hospital stay, days & Median (range) & $22(9-123)$ & $24.5(9-56)$ & 0.896 \\
\hline
\end{tabular}

CEA: Carcinoembryonic antigen; CA19-9: carbohydrate antigen 19-9; CDC: Clavien-Dindo classification. Bold values indicate statistical significance.

Patients with prolonged PT-INR had significantly worse OS than those with normal PT-INR in the survival curve analysis, the median OS in patients with prolonged PT-INR being significantly shorter than that of patients with normal PT-INR (17 vs. 33 months, respectively; $p=0.003$ ) (Figure 1B). Interestingly, when other patient characteristics were compared between patients with normal and prolonged PTINR, male sex, CA19-9, and large tumor size were found to be significantly associated with prolonged PT-INR (Table V). However, prolonged PT-INR was not significantly associated with surgical outcomes such as intraoperative blood loss and operative time. Postoperative bleeding events requiring transfusion of red blood cells such as rupture of a pseudoaneurysm $(n=1)$, bleeding from drain $(n=3)$, bleeding from anastomosis $(n=1)$, anemia progression $(n=1)$ occurred in six patients (three with normal PT-INR and three with prolonged PT-INR). Postoperative thrombosis was detected in four patients (portal thrombosis in two, deep venous thrombosis in one, and renal infarction in one), all of whom had normal PT-INR. Although prolonged PT-INR was not significantly associated with postoperative bleeding events and thrombosis $(p=0.056$ and $p=$ not available), bleeding 
complications in three patients with prolonged PT-INR, namely bleeding from a drain, bleeding from anastomosis, and anemia progression in one patient each, may have been related to their coagulation disorders (Table V).

\section{Discussion}

In this study, coagulation disorders characterized by prolonged PT-INR were associated with worse outcomes in patients with PDAC who had undergone curative resection. Thus, we have here identified prolonged PT-INR as a novel marker of poor prognosis in patients with PDAC. The coagulation disorders that are often detected in patients with cancer are usually subclinical, even when coagulation tests are abnormal (15). The PT is a simple test for detecting and diagnosing bleeding or excessive clotting disorders and the international normalized ratio (INR) is a means of standardizing the PT.

Although coagulation disorders in individuals with cancer are multifactorial in origin, several studies have reported the important role of coagulation activity in cancer progression (16, 17). Vitamin K, a fat-soluble vitamin, is necessary for the blood coagulation cascade. Vitamin $\mathrm{K}$ activates coagulation factors II, VII, IX, X, which promote coagulation. In vitamin K deficiency, the blood concentration of protein induced by vitamin $\mathrm{K}$ absence II (PIVKA-II), a precursor of factor II, increases (18). PIVKA-II has been used as a tumor marker, especially for hepatocellular carcinoma. Although patients with malignant tumors other than hepatocellular carcinoma rarely have a high serum PIVKA-II concentration, a high PIVKA-II level has been reported in association with lung, stomach, colon, and ovarian cancer (19-22). The serum concentration of PIVKA-II has also been reported to be higher in patients with pancreatic cancer than in those with benign pancreatic diseases; thus, it may be useful as a tumor biomarker (23). Vitamin K deficiency may be associated with prognosis of patients with pancreatic cancer.

Furthermore, coagulation disorders associated with cancer are reportedly caused by activation of tissue factor (TF) in tumor tissue and activation of MET oncogene (4). TF is recognized as playing an important role in this coagulation process. It is a transmembrane receptor for factor VII/VIIa (FVII/VIIa). The TF:FVIIa complex is called the 'extrinsic' pathway because an exogenous agent is required for activation of clotting factors in plasma (24). The TF:FVIIa complex is the key initiator of the coagulation protease cascade, activating both FIX to FIXa and FX to FXa (24). The PT is determined by adding exogenous TF to plasma and is used to assess the activity of the extrinsic pathway. TF and its ligand FVIIa have been shown to play a role in tumor metastasis. The TF:FVIIa complex has been reported to influence tumor metastasis through tumor angiogenesis, inhibition of apoptosis and induces intracellular changes such as cell survival (25). The TF:FVIIa complex has been shown to be an independent risk factor for hepatic metastasis in patients with colon cancer and to play a role in ovarian cancer invasion and metastasis (26-28). Thus, several studies have recently reported on the involvement of the TF:FVIIa complex in cancer progression. To our knowledge, although TF is not known to be associated with progression of pancreatic cancer, TF in patients with pancreatic cancer may act to contribute to prolonged PTINR, leading to worse prognosis. The underlying mechanism for prolonged PT-INR in patients with PDAC and worse outcomes requires elucidation by further investigation.

The first limitation of this study is that it was retrospective. A second limitation is the validity of cut-off values for coagulation factors. We based the cut-off value for each clotting factor on normal values for our Institution. Other institutions may have different cut-off values, which might lead to different results. A third limitation is that some coagulation factors, such as Ddimers and fibrinogen, were not measured in all study patients and are possibly prognostic markers. A large prospective study is required to further investigate the role of coagulation factors as prognostic factors and their cut-off values.

In conclusion, a prolonged PT-INR might be a simple and novel predictor of prognosis in patients who have undergone curative resection of PDAC. Preoperative normalization of coagulation activity may be an alternative strategy for improving the prognosis of patients with PDAC.

\section{Conflicts of Interest}

All Authors of this article have no conflicts of interest to declare.

\section{Authors' Contributions}

Conceptualization: Kazuki Matsumura. Methodology: Kazuki Matsumura, Hiromitsu Hayashi. Formal analysis and investigation: Kazuki Matsumura, Hiromitsu Hayashi. Data curation: Kazuki Matsumura, Norio Uemura, Fumimasa Kitamura, Yusuke Nakao, Toshihiko yusa, Rumi Itoyama. Writing original draft preparation: Kazuki Matsumura. Writing, review and editing: Hiromitsu Hayashi. Supervision: Hideo Baba. All Authors approved the final draft of the article.

\section{Acknowledgements}

The Authors thank Dr. Trish Reynolds, MBBS, FRACP, from Edanz Group (www.edanzediting.com/ac) for editing a draft of this article.

\section{References}

1 Vincent A, Herman J, Schulick R, Hruban RH and Goggins M: Pancreatic cancer. Lancet 378(9791): 607-620, 2011. PMID: 21620466. DOI: 10.1016/S0140-6736(10)62307-0

2 Gillen S, Schuster T, Meyer Zum Büschenfelde C, Friess H and Kleeff J: Preoperative/neoadjuvant therapy in pancreatic cancer: A systematic review and meta-analysis of response and resection percentages. PLOS Med 7(4): e1000267, 2010. PMID: 20422030. DOI: 10.1371/journal.pmed.1000267

3 Lyman GH and Khorana AA: Cancer, clots and consensus: New understanding of an old problem. J Clin Oncol 27(29): 48214826, 2009. PMID: 19752337. DOI: 10.1200/JCO.2009.22.3032 
4 Khorana AA and Fine RL: Pancreatic cancer and thromboembolic disease. Lancet Oncol 5(11): 655-663, 2004. PMID: 15522652. DOI: $10.1016 / \mathrm{S} 1470-2045(04) 01606-7$

5 Godinho J, Casa-Nova M, Moreira-Pinto J, Simões P, Paralta Branco F, Leal-Costa L, Faria A, Lopes F, Teixeira JA and Passos-Coelho JL: ONKOTEV score as a predictive tool for thromboembolic events in pancreatic cancer - a retrospective analysis. Oncologist 25(2): e284-e290, 2020. PMID: 32043787. DOI: 10.1634/theoncologist.2019-0510

6 Khorana AA, Kuderer NM, Culakova E, Lyman GH and Francis $\mathrm{CW}$ : Development and validation of a predictive model for chemotherapy-associated thrombosis. Blood 111(10): 4902-4907, 2008. PMID: 18216292. DOI: 10.1182/blood-2007-10-116327

7 Iguchi T, Sugimachi K, Mano Y, Kono M, Kagawa M, Nakanoko T, Uehara H, Sugiyama M, Ota M, Ikebe M, Morita M4 and Toh Y: The preoperative prognostic nutritional index predicts the development of deep venous thrombosis after pancreatic surgery. Anticancer Res 40(4): 2297-2301, 2020. PMID: 32234929. DOI: 10.21873/anticanres.14195

8 Zhang XP and Cheng SQ: ASO Author Reflections: Preoperative hypercoagulability predicts poor prognosis in hepatocellular carcinoma patients with microvascular invasion after hepatectomy. Ann Surg Oncol 26: 806-807, 2019. PMID: 31654157. DOI: $10.1245 / \mathrm{s} 10434-019-07986-5$

9 Oberhoff C, Rollwagen C, Tauchert AM, Hoffmann O, Winkler $\mathrm{UH}$ and Schindler AE: Perioperative development of a thrombogenic risk profile in patients with carcinoma of breast: A cause of increased thrombosis. Eur J Gynaecol Oncol 21(6): 560-568, 2000. PMID: 11214610.

10 Mei Y, Liu H, Sun X, Li X, Zhao S and Ma R: Plasma fibrinogen level may be a possible marker for the clinical response and prognosis of patients with breast cancer receiving neoadjuvant chemotherapy. Tumour Biol 39(6): 1010428317700002, 2017. PMID: 28621233. DOI: 10.1177/1010428317700002

11 Oya M, Akiyama Y, Okuyama $\mathrm{T}$ and Ishikawa H: High preoperative plasma D-dimer level is associated with advanced tumor stage and short survival after curative resection in patients with colorectal cancer. Jpn J Clin Oncol 31(8): 388-394, 2001. PMID: 11574632. DOI: 10.1093/jjco/hye075

12 Tas F, Kilic L, Serilmez M, Keskin S, Sen F and Duranyildiz D: Prognostic significance of coagulation assays in lung cancer. Respir Med 107(3): 451-457, 2013. PMID: 23200643. DOI: 10.1016/j.rmed.2012.11.007

13 Nimura, Y, Nagino M, Takao S, Takada T, Miyazaki K, Kawarada Y, Miyagawa S, Yamaguchi A, Ishiyama S, Takeda Y, Sakoda K, Kinoshita T, Yasui K, Shimada H and Katoh H: Standard versus extended lymphadenectomy in radical pancreatoduodenectomy for ductal adenocarcinoma of the head of the pancreas: long-term results of a Japanese multicenter randomized controlled trial. J Hepatobiliary Pancreat Sci 19(3): 230-241, 2012. PMID: 22038501. DOI: $10.1007 / \mathrm{s} 00534-011-0466-6$

14 Dindo D, Demartines N and Clavien PA: Classification of surgical complications: A new proposal with evaluation in a cohort of 6336 patients and results of a survey. Ann Surg 240(2): 205-213, 2004. PMID: 15273542. DOI: 10.1097/01.sla.0000133083.54934.ae

15 Falanga A: Thrombophilia in cancer. Semin Thromb Hemost 31(1): 104-110, 2005. PMID: 15706482. DOI: 10.1055/s-2005-863812

16 Lima LG and Monteiro RQ: Activation of blood coagulation in cancer: Implications for tumour progression. Biosci. Rep 33(5): e00064, 2013. PMID: 23889169. DOI: 10.1042/BSR20130057
17 Ruf W, Rothmeier AS and Graf C: Targeting clotting proteins in cancer therapy - progress and challenges. Thromb Res 140: S1-S7, 2016. PMID: 27067961. DOI: 10.1016/S0049-3848(16)30090-1

18 Dong R, Wang N, Yang Y, Ma L, Du Q, Zhang W, Tran AH, Jung H, Soh A, Zheng Y and Zheng S: Review on vitamin K deficiency and its biomarkers: Focus on the novel application of PIVKA-II in clinical practice. Clin Lab 64(4): 413-424, 2018. PMID: 29739078. DOI: 10.7754/Clin.Lab.2017.171020

19 Takahashi Y, Inoue $\mathrm{T}$ and Fukusato T: Protein induced by vitamin $\mathrm{K}$ absence or antagonist II-producing gastric cancer. World J Gastrointest Pathophysiol 1(4): 129-136, 2010. PMID: 21607153. DOI: 10.4291/wjgp.v1.i4.129

20 Nasu M, Soma T, Fukushima H, Kudo K and Matsubara O: Hepatoid carcinoma of the lung with production of alphafetoprotein and abnormal prothrombin: An autopsy case report. Mod Pathol 10(10): 1054-1058, 1997. PMID: 9346187.

21 Miyashita K, Nagasaka A, Nakanishi M, Kudo T, Wakahama O, Nishikawa S, Higuchi A and Sato H: An alpha-fetoprotein and PIVKA-II producing carcinoma of the colon: report of a case. Nippon Shokakibyo Gakkai Zasshi 97(12): 1480-1486, 2000. PMID: 11193493.

22 Senzaki H, Kiyozuka Y, Mizuoka H, Yamamoto D, Ueda S, Izumi $\mathrm{H}$ and Tsubura A: An autopsy case of hepatoid carcinoma of the ovary with PIVKA-II production: Immunohistochemical study and literature review. Pathol Int 49(2): 164-169, 1999. PMID: 10355972. DOI: 10.1046/j.1440-1827.1999.00840.x

23 Tartaglione S, Pecorella I, Zarrillo SR, Granato T, Viggiani V, Manganaro L, Marchese C, Angeloni A and Anastasi E: Protein induced by Vitamin $\mathrm{K}$ absence II (PIVKA-II) as a potential serological biomarker in pancreatic cancer: A pilot study. Biochem Med (Zagreb) 29(2): 020707, 2019. PMID: 31223261. DOI: 10.11613/BM.2019.020707

24 Mackman N: The role of tissue factor and factor VIIa in Hemostasis. Anesth Analg 108(5): 1447-1452, 2009. PMID: 19372318. DOI: 10.1213/ane.0b013e31819bceb1

25 Versteeg HH, Spek CA, Peppelenbosch MP and Richel DJ: Tissue factor and cancer metastasis: The role of intracellular and extracellular signaling pathways. Mol Med 10(1-6): 6-11, 2004. PMID: 15502877. DOI: 10.2119/2003-00047.versteeg

26 Versteeg HH, Spek CA, Slofstra SH, Diks SH, Richel DJ and Peppelenbosch MP: FVIIa:TF Induces cell survival via $\mathrm{G}_{12} / \mathrm{G}_{13^{-}}$ dependent Jak/STAT activation and $\mathrm{Bcl}_{\mathrm{XL}}$ production. Circ Res 94(8): 1032-1040, 2004. PMID: 15016732. DOI: 10.1161/01. RES.0000125625.18597.AD

27 Seto S, Onodera H, Kaido T, Yoshikawa A, Ishigami S, Arii S and Imamura M: Tissue factor expression in human colorectal carcinoma: Correlation with hepatic metastasis and impact on prognosis. Cancer 88(2): 295-301 2000. PMID: 10640960. DOI: 10.1002/(sici) 1097-0142(20000115)88:2<295::aid-cncr8>3.0.co;2-u

28 Ma Z, Zhang T, Wang R, Cheng Z, Xu H, Li W, Wang Y and Wang X: Tissue factor-factor VIIa complex induces epithelial ovarian cancer cell invasion and metastasis through a monocytedependent mechanism. Int J Gynecol Cancer 21(4): 616-624, 2011. PMID: 21543928. DOI: 10.1097/IGC.0b013e3182150e98

Received May 8, 2020

Revised June 18, 2020

Accepted June 22, 2020 